\title{
Answer to Quiz (BIRDEM Med J 2011; 1(1): 54)
}

\section{Safdar AMB}

(Birdem Med J 2012; 2(1): 70)

Fig.-1 : Salmon colored papules \& nodules over the face in a butterfly distribution particularly in nasolabial fold.

Fig.-2: Showed irregularly shaped evenly pigmented brown macule over right forearm.

Fig.-3: X-ray skull lateral view revealed calcification on the calvarium.

Fig.-4: CT scan of Head revealed multiple calcified ependymal nodules.

The likely diagnosis is Tuberous Sclerosis Complex (TSC).

Tuberous sclerosis complex (TSC) is an inherited neurocutaneous disorder that is characterized by pleomorphic features involving many organ systems, including multiple benign hamartomas of the brain, eyes, heart, lung, liver, kidney, and skin. It is an autosomal dominant genetic disorder with highly variable expression \& severity. Mutations in either one of the two separate genes TSC1 (chromosome 9q34 \& encodes hamartin protein) and TSC2 (chromosome 16p13.3 \& encodes the tuberin protein) is responsible for the disease process. Incidence is about 1 in 5,000 to 10,000 live births. It affects both sex equally with no racial predilection. TSC can present at any age. The classic TSC diagnostic triad (Vogt's triad) i.e. seizures, mental retardation \& facial angiofibromas, is present in less than one-third of patients with TSC. According to the new diagnostic criteria by the National Tuberous Sclerosis Association (USA), TSC has been classified as Definite, Probable and Possible TSC on the basis of presence of some major and / or minor features. The diagnosis of TSC is made clinically.
All patients with suspected TSC should undergo CT / MRI of the Brain, Skull X-Ray, Renal Ultrasound \& Echocardiogram for confirmation \& also to see extent of the disease. Molecular genetic testing to find out the mutations is only required in uncertain or questionable cases, for prenatal diagnosis, and for screening family members of an affected individual.

Treatment goal is to reduce morbidity and to prevent complications. It includes

- MTOR Kinase Inibitor (Everolimus) which is indicated for subependymal giant cell astrocytoma (SEGA) associated with tuberous sclerosis (TS) that cannot be treated with surgery.

- Anticonvulsants.

- Surgery or intervention.

Death in these patients may be due to

- Sudden unexplained death in epilepsy or related to an accident involving a seizure.

- Critical hydrocephalus from undiagnosed giant cell astrocytoma.

- Cardiac arrhythmia.

- Hemorrhagic complications of renal AMLs.

- Rupture of occult arterial aneurysms.

Prognosis is variable. Higher numbers of tubers, earlier onset and intractability of seizures, and infantile spasms are associated with worse cognitive and behavioral outcomes. Cardiac lesions almost always regress spontaneously. Pulmonary and renal lesions affect prognosis on the basis of their extent and severity. 